\title{
Factors affecting bacteriology of hand sepsis in South Africa
}

\author{
M van der Vyver, (i) A Maderee (iD \\ Department of Plastic and Reconstructive Surgery, Inkosi Albert Luthuli Central Hospital, South Africa
}

Corresponding author, email: marietavdv@gmail.com

Background: Hand sepsis is a common cause of morbidity. The study was conducted in the KwaZulu-Natal province of South Africa, investigating the bacteriological profile and aetiology of hand sepsis, aiming at optimising empiric antibiotic therapy.

Methods: This is a descriptive study of 120 patients who presented to the plastic surgery department of Inkosi Albert Luthuli Central Hospital with hand sepsis, from January 2017 to April 2019, that required surgical drainage. Data recorded included: aetiology of sepsis, underlying comorbidities, length of hospital stay, and outcome. A pus swab was taken during surgical drainage for microscopy, culture and sensitivity (MCS) analysis. The relationship between clinical factors and microbiological findings was analysed.

Results: The predominant bacterial strains cultured from infected patients, excluding methicillin-resistant Staphylococcus aureus, were Staphylococcus aureus (80.8\%) and Streptococcus spp. (20.0\%), mostly due to spontaneous onset infection $(58.3 \%)$. Cultures of the more problematic Streptococcus spp. were more frequently associated in patients with diabetes mellitus $(60.0 \%)$, penetrating trauma $(41.9 \%)$, or blunt trauma $(31.3 \%)$. The diabetes group in isolation had the longest median hospital stay of $8(5-15)$ days, the most need for multiple procedures $(60.0 \%)$, amputations $(30.0 \%)$, and skin grafts $(20.0 \%)$. The most effective antimicrobial drug particularly in mixed cultures was amoxicillin-clavulanic acid.

Conclusion: Staphylococcus aureus infection was the most common. Streptococcus spp. occurred more frequently in patients with traumatic injury and diabetes mellitus. The latter required further surgical procedures and an increased hospital stay. Amoxicillin-clavulanic acid is the preferred empiric antimicrobial agent.

Keywords: hand sepsis, bacteriology, optimising empiric antibiotic therapy

\section{Introduction}

Hand infections are a common occurrence, often necessitating prompt medical interventions. Early microbiological diagnosis aids in the appropriate antibiotic therapy, thereby reducing morbidity. However, optimisation of antibiotic therapy is still a challenge in the treatment and management of infections. Despite advancements in medical interventions, management of hand infections is still a concern. Increases in antibiotic-resistant microbes, delayed diagnosis, and socio-economic constraints of patients are some of the factors that hinder the appropriate treatment of hand infections. 1 Among these factors, the increasing resistance of bacteria against antimicrobial agents is of primary concern. Hence, detailed investigation of microbial spectrums along with their sensitivities to specific antimicrobial agents may help in overcoming the recurrence of infections and other associated complications. 2 The cultured microbes from hand infections usually show variations in different populations, with different risk factors. 3 The most common mechanism of hand infections is trauma, and the most common causative organism is Staphylococcus aureus ${ }^{4-6}$ with Streptococcus spp. the next most frequent., $, 5,7-10$ Literature suggests that immunosuppression influences the type of bacteria cultured and its sensitivity to antibiotics. ${ }^{4}$ It has been reported in a local study that HIV positive patients were more likely to culture gram-negative organisms, that were resistant to
Cloxacillin. ${ }^{5}$ Other organisms reported in hand infections include Streptococcus spp., gram-negative organisms and viruses. ${ }^{4,10}$ Several studies reported an increase in the occurrence of methicillin-resistant Staphylococcus aureus (MRSA) in hand sepsis, most of which have been attributed to nosocomial and community-acquired infections.4,8,9

Epidemiological studies revealed that manual workers are more prone to hand infections due to a lack of protection to their hands and the use of inferior types of equipment owing to their poor socio-economic background. ${ }^{1}$ Specific groups of patients with comorbidities like diabetes and HIV are susceptible to polymicrobial hand infections due to decreased immunity, as are patients on corticosteroid therapy and intravenous drug users. ${ }^{4,7,11,12}$

Many patients in KwaZulu-Natal are potentially immunocompromised due to either HIV or diabetes mellitus. ${ }^{13}$ Hence, microbial investigations and antibiotic therapy are important components of the management of hand sepsis. However, formal microscopy, culture and sensitivity (MCS) analysis at the time of presentation takes over 48 hours and cannot guide the initial choice of antibiotic therapy. Therefore, knowing the probable bacteria and their antimicrobial susceptibility is key to effective therapy. This study aims to analyse the relationship between the clinical factors of hand infections, and their bacteriological spectrum to guide the choice of optimal empirical antimicrobial therapy. 


\section{Methods}

One hundred and twenty patients had surgical drainage of hand infections by the plastic and reconstructive surgery department at Inkosi Albert Luthuli Central Hospital (IALCH) from January 2017 until April 2019. Patients who did not require surgical drainage of pus or patients in whom a pus swab was not collected during surgical drainage were excluded.

A standard format was used to collect information on demographics, hand dominance, underlying comorbidities (diabetes and HIV status), mechanism of injury and presentation of the hand injury (affected hand, circulation). Pus swabs were taken during drainage, and subjected to MCS analysis. All laboratory reports were examined, and identities of etiologic organisms were recorded. Antimicrobial sensitivity analysis was conducted by the laboratory, following standard protocol. ${ }^{14}$

Descriptive statistics are presented by way of means and standard deviations (SDs), interquartile ranges (IQRs), and categorical variables through frequencies with percentages. Analyses were performed with Microsoft Excel.

\section{Results}

One hundred and twenty patients presented with hand sepsis requiring surgical drainage during the study period. The demographic, clinical and etiological profile of the cohort of 120 patients is shown in Table I. One patient had both hands affected. Fingers were affected in 94 patients $(79.2 \%)$, hand palmar surface in $26(21.7 \%)$, hand dorsal surface in 24 $(20.0 \%)$, forearm volar in two $(1.7 \%)$, and forearm dorsum in three $(2.5 \%)$ patients.

Four patients did not show any growth of microorganisms. Twelve different microbial species were cultured. The most commonly isolated microorganisms were Staphylococcus aureus and Streptococcus spp. Ninety-eight patients (81\%) had a culture of Staphylococcus aureus (one was MRSA), or in combination with Streptococcus pyogenes, of which 89 patients cultured Staphylococcus aureus alone. Eight patients had mixed cultures of Streptococcus pyogenes and Staphylococcus aureus. Twenty-four patients (20.0\%) had a culture of Streptococcus spp., either alone or in combination with other bacterial species. Enterobacter cloacae, Escherichia coli and Klebsiella pneumonia were each cultured on a single occasion.

The most cultured microorganism in the 70 patients with spontaneous infections was Staphylococcus aureus (88.6\%), followed by Streptococcus spp. (5.7\%). Polymicrobial cultures were present in $2.9 \%$ of patients with spontaneous infection. The dominant microorganism in patients with penetrating trauma was Staphylococcus aureus (71.0\%), followed by Streptococcus spp. (41.9\%), mixed cultures (19.4\%), MRSA (3.2\%) and Escherichia coli (3.2\%). Most of the patients with blunt trauma cultured Staphylococcus aureus (68.8\%), followed by Streptococcus spp. (31.3\%) and polymicrobial cultures $(18.8 \%)$. Noticeably, $60 \%$ of patients with diabetes mellitus also cultured Streptococcus spp. Two patients sustained insect bites, of which one patient cultured Staphylococcus aureus (50.0\%), and another Streptococcus pyogenes $(50.0 \%)$. Only one patient had a human bite and cultured Streptococcus constellatus. The finding is contrary to an earlier study where polymicrobial cultures were reported in patients with human bites. ${ }^{15}$

\begin{tabular}{|c|c|c|}
\hline \multirow[t]{2}{*}{ Patient characteristic } & \multicolumn{2}{|c|}{$n=120$} \\
\hline & Median & IQR \\
\hline \multirow[t]{2}{*}{ Age in years } & 32.0 & $22-39$ \\
\hline & Number & Per cent \\
\hline \multicolumn{3}{|l|}{ Gender } \\
\hline Male & 93 & 77.5 \\
\hline Female & 27 & 22.5 \\
\hline \multicolumn{3}{|l|}{ Employment status } \\
\hline Manual labour & 26 & 21.7 \\
\hline Non-manual labour & 92 & 76.7 \\
\hline Others & 2 & 1.7 \\
\hline \multicolumn{3}{|l|}{ Hand dominance } \\
\hline Left-handed & 12 & 10.0 \\
\hline Right-handed & 105 & 87.5 \\
\hline Ambidextrous & 1 & 0.8 \\
\hline Unconfirmed & 2 & 1.7 \\
\hline Dominant hand affected & 63 & 52.5 \\
\hline Right hand affected & 60 & 50.0 \\
\hline \multicolumn{3}{|l|}{ Etiology } \\
\hline Spontaneous & 70 & 58.4 \\
\hline Penetrating trauma & 31 & 25.8 \\
\hline Blunt trauma & 16 & 13.3 \\
\hline Human bite & 1 & 0.8 \\
\hline Insect/spider bite & 2 & 1.7 \\
\hline \multicolumn{3}{|l|}{ Hand circulation } \\
\hline Normal & 113 & 94.2 \\
\hline Decreased & 4 & 3.3 \\
\hline Absent & 3 & 2.5 \\
\hline \multicolumn{3}{|l|}{ Comorbidities } \\
\hline Diabetes mellitus & 10 & 8.3 \\
\hline $\mathrm{HIV}+$ status & 28 & 23.3 \\
\hline DM and HIV & 1 & 0.8 \\
\hline
\end{tabular}

In diabetic patients with hand sepsis, the most commonly cultured organism was Streptococcus spp. (60.0\%), followed by Staphylococcus aureus (30.0\%). Forty per cent of diabetic patients cultured multiple microorganisms and $10.0 \%$ were without any growth of microorganisms. The most cultured microorganism in patients with HIV was Staphylococcus aureus (85.7\%), followed by Streptococcus spp. (14.3\%), and Escherichia coli (3.6\%). Patients with a mixed culture, and without a positive culture for microorganisms, account for $7.1 \%$ and $3.6 \%$, respectively. Only one patient had both HIV and diabetes, and cultured Staphylococcus aureus.

Table II details the association of etiological factors and comorbidities with microbial cultures. Diabetic patients with spontaneous onset of infection showed a positive culture for 
Table II: Association of etiological factors and comorbidities with microbial culture

\begin{tabular}{|c|c|c|c|c|c|c|}
\hline \multirow{3}{*}{$\begin{array}{l}\text { Comorbid } \\
\text { condition }\end{array}$} & \multicolumn{6}{|c|}{ Etiological factors } \\
\hline & \multicolumn{2}{|r|}{ Spontaneous $n=70$} & \multicolumn{2}{|r|}{ Penetrating trauma $n=\mathbf{3 1}$} & \multicolumn{2}{|r|}{ Blunt trauma $n=16$} \\
\hline & $\mathrm{n}$ & Organism $(n)$ & $\mathrm{n}$ & Organism $(n)$ & $\mathrm{n}$ & Organism $(n)$ \\
\hline $\begin{array}{l}\mathrm{DM} \\
n=10\end{array}$ & 7 & $\begin{array}{c}\text { S. aureus } 2 \\
\text { Str. anginosus } 1 \\
\text { Str. intermedius } 1 \\
\text { Str. pyogenes \& S. aureus } 1 \\
\text { Mixed growth } 1 \\
\text { No identifiable growth } 1\end{array}$ & 2 & $\begin{array}{c}\text { Str. anginosus } 1 \\
\text { E. cloacae, K. pneumonia \& Str. } \\
\text { sanguinis } 1\end{array}$ & 1 & $\begin{array}{l}\text { Str. mitis/oralis \& Str. } \\
\text { constellatus } 1\end{array}$ \\
\hline $\begin{array}{l}\text { HIV+ status } \\
n=28\end{array}$ & 18 & $\begin{array}{l}\text { S. aureus } 16 \\
\text { Str. pyogenes } 1 \\
\text { No growth } 1\end{array}$ & 6 & $\begin{array}{c}\text { S. aureus } 2 \\
\text { Str. pyogenes \& } S \text {. aureus } 2 \\
\text { Str. pyogenes } 1 \\
\text { E. coli } 1\end{array}$ & 4 & S. aureus 4 \\
\hline $\begin{array}{l}\text { DM and } \\
\text { HIV }\end{array}$ & 1 & S. aureus 1 & & & & \\
\hline
\end{tabular}

DM - diabetes mellitus, HIV - human immunodeficiency virus

\section{Table III: Antimicrobial sensitivity for the dominant microorganisms}

\begin{tabular}{|c|c|c|c|c|c|c|c|c|c|c|c|c|c|c|c|}
\hline \multirow{3}{*}{ Antimicrobial agents } & \multicolumn{15}{|c|}{ Microorganisms } \\
\hline & \multicolumn{5}{|c|}{ *MRSA } & \multicolumn{5}{|c|}{ Staphylococcus aureus } & \multicolumn{5}{|c|}{ Streptococcus spp } \\
\hline & $n$ & $\mathbf{S}$ & $\%$ & $\mathbf{R}$ & $\%$ & $n$ & $\mathbf{S}$ & $\%$ & $\mathbf{R}$ & $\%$ & $n$ & $\mathbf{S}$ & $\%$ & $\mathbf{R}$ & $\%$ \\
\hline Amoxicillin-clavulanic acid & - & - & - & - & - & 30 & 30 & 100 & 0 & 0 & 12 & 12 & 100 & 0 & 0 \\
\hline Penicillin/ampicillin & - & - & - & - & - & 1 & 0 & 0 & 1 & 100 & 24 & 24 & 100 & 0 & 0 \\
\hline Cloxacillin & 1 & 0 & 0 & 1 & 100 & 96 & 96 & 100 & 0 & 0 & - & - & - & - & - \\
\hline Clindamycin & - & - & - & - & - & 79 & 79 & 100 & 0 & 0 & - & - & - & - & - \\
\hline Erythromycin/azithromycin & - & - & - & - & - & 59 & 59 & 100 & 0 & 0 & - & - & - & - & - \\
\hline Cefazolin & - & - & - & - & - & 31 & 31 & 100 & 0 & 0 & 1 & 1 & 100 & 0 & 0 \\
\hline Trimethoprim-sulfamethoxazole & - & - & - & - & - & 5 & 5 & 100 & 0 & 0 & - & - & - & - & - \\
\hline Vancomycin & 1 & 1 & 100 & 0 & 0 & 1 & 1 & 100 & 0 & 0 & - & - & - & - & - \\
\hline
\end{tabular}

*MRSA - methicillin-resistant Staphylococcus aureus

$n-$ total number of cultures

\begin{tabular}{|c|c|c|c|c|c|c|c|c|c|c|c|c|}
\hline \multirow[t]{2}{*}{ Patient characteristics } & \multicolumn{2}{|c|}{ Total patients } & \multicolumn{2}{|c|}{${ }^{1}$ Discharged } & \multicolumn{2}{|c|}{$\begin{array}{l}{ }^{2} \text { Multiple } \\
\text { procedures }\end{array}$} & \multicolumn{2}{|c|}{ Amputation } & \multicolumn{2}{|c|}{$\begin{array}{l}\text { Skin graft/ } \\
\text { flap }\end{array}$} & \multicolumn{2}{|c|}{${ }^{3} \mathbf{L O H}$} \\
\hline & $n$ & $\%$ & $n$ & $\%$ & $n$ & $\%$ & $n$ & $\%$ & $n$ & $\%$ & Median & IQR \\
\hline Total sample & 120 & 100.0 & 95 & 79.2 & 25 & 20.8 & 5 & 4.2 & 10 & 8.3 & 3 & $1.5-6$ \\
\hline Diabetes mellitus & 10 & 8.3 & 4 & 40.0 & 6 & 60.0 & 3 & 30.0 & 2 & 20.0 & 8 & $5-15$ \\
\hline HIV & 28 & 23.3 & 22 & 78.6 & 3 & 10.7 & 1 & 3.6 & 2 & 7.1 & 4 & $1.5-6.5$ \\
\hline Penetrating trauma & 31 & 25.8 & 25 & 80.6 & 6 & 19.4 & 1 & 3.2 & 3 & 9.7 & 3 & $1-5$ \\
\hline Blunt trauma & 16 & 13.3 & 14 & 87.5 & 2 & 12.5 & 1 & 6.3 & 1 & 6.3 & 2 & $0.5-4$ \\
\hline $\begin{array}{l}\text { With pure culture of } \\
\text { Staphylococcus aureus }\end{array}$ & 89 & 74.2 & 73 & 82.0 & 16 & 18.0 & 1 & 1.1 & 6 & 6.7 & 3 & $1-5$ \\
\hline $\begin{array}{l}\text { With pure culture of } \\
\text { Streptococcus spp. }\end{array}$ & 15 & 12.5 & 11 & 73.3 & 4 & 26.7 & 1 & 6.7 & 2 & 13.3 & 5 & $2-9$ \\
\hline $\begin{array}{l}\text { Any culture containing } \\
\text { Staphylococcus aureus }\end{array}$ & 98 & 81.7 & 81 & 82.7 & 17 & 17.3 & 1 & 1.0 & 6 & 6.1 & 3 & $1-5$ \\
\hline $\begin{array}{l}\text { Any culture containing } \\
\text { Streptococcus spp. }\end{array}$ & 24 & 20.0 & 18 & 75.0 & 6 & 25.0 & 2 & 8.3 & 3 & 12.5 & 5 & $2-7.5$ \\
\hline
\end{tabular}

1 - Discharged after single incision and drainage, 2 - Multiple procedures required, 3 - Length of hospital stay 
Staphylococcus aureus and Streptococcus spp. Among the 28 HIV positive patients, Staphylococcus aureus was the dominant bacterial strain.

Table III shows the antimicrobial sensitivities. Staphylococcus aureus and Streptococcus spp. were universally sensitive to amoxicillin-clavulanic acid. Only one MRSA which was sensitive to vancomycin was cultured, probably due to the infection being acquired outside a hospital environment. Penicillin/ampicillin sensitivity was performed only once for Staphylococcus aureus due to the very high background resistance from this organism but tested 100\% sensitive against Streptococcus spp. Staphylococcus aureus showed sensitivity to cloxacillin, clindamycin, erythromycin/azithromycin, cefazolin and trimethoprim-sulfamethoxazole. Enterobacter cloacae was found sensitive to ciprofloxacin, while $E$. coli was sensitive to amoxicillin-clavulanic acid. Amoxicillin-clavulanic acid was found to be the single most effective antibacterial agent against the predominant bacteria Staphylococcus aureus and Streptococcus spp.

Patients with diabetes required a higher rate of multiple procedures $(60.0 \%)$, amputation $(30.0 \%)$, and skin graft/ flap $(20.0 \%)$ as a result of sepsis (Table IV). Patients with diabetes mellitus or those with any positive culture for Streptococcus spp. had the longest duration of hospital stay, with 8 (IQR 5-15) and 5 (IQR 2-7.5) days, respectively (Table IV).

\section{Discussion}

Identification of predisposing factors are important for all patients with hand sepsis. Furthermore, positive associations of the microbial spectrum with these predisposing factors have clinical significance in choosing appropriate antibiotics by the physician prior to microbial test results. ${ }^{16}$ Previous studies suggested that the most common mechanism of hand infections is trauma. ${ }^{5,6,17}$ Contrary to these studies, present findings revealed spontaneous onset infection as the more common cause as opposed to those following trauma. ${ }^{18}$ In the present study, most of the affected patients were males, which is consistent with earlier studies. ${ }^{19}$ The right hand showed preponderance over the left hand in terms of dominance, although no association was found between hand dominance and the pattern of injury. Manual labourers were found to be more prone to risk of injury, exposing them to microbial infections.

Staphylococcus aureus was the most predominant species isolated of all bacteria. Present findings revealed that more than $80.8 \%$ of patients cultured Staphylococcus aureus, which is consistent with other reports. ${ }^{4,5,7-10}$ Another frequent species was Streptococcus spp. (20.0\%). Other bacteria that were isolated include Enterobacter cloacae, Escherichia coli, Klebsiella pneumonia and MRSA. It may be mentioned that MRSA, Enterobacter cloacae, Escherichia coli and Klebsiella pneumonia were rarely detected in the studied population. Different bacterial strains were found associated with specific etiological factors and underlying comorbidities. It was observed that patients with spontaneous infection were more susceptible to infection by Staphylococcus aureus, while patients with penetrating trauma, blunt trauma or insect bites were susceptible to both Staphylococcus aureus and Streptococcus spp. Previous studies have shown that patients with diabetes mellitus and HIV patients are at higher risk of polymicrobial cultures, which might be attributed to impaired immune functions. ${ }^{4-7,20}$ The positive culture rate of patients with comorbidities and polymicrobial cultures was $16.2 \%$. There was also a positive association between certain comorbidities and specific strains. Patients with HIV, for example, had higher positive culture rates for Staphylococcus aureus compared to other bacterial strains, whereas patients with diabetes mellitus had a higher incidence of Streptococcus spp. culture.

Diabetics had a higher rate of amputation $(30.0 \%)$ or multiple procedures $(60.0 \%)$ and, not surprisingly, a longer duration of hospital stay by an average of 3 days. The findings corroborate those of an earlier study on outcomes following HIV and diabetic hand infections. ${ }^{21,22}$ The higher rates of diabetic patients with severe outcomes would be aggravated by poorly controlled diabetes and prevalence of polymicrobial cultures. ${ }^{23}$ The amputation rate for a patient with trauma injury was $4.0 \%$. The results are consistent with some of the earlier findings on amputation rates of upper extremities for containment of infections..$^{24,25}$ Furthermore, patients with a positive culture for Streptococcus spp. were inclined to undergo multiple procedures, amputation, or skin grafts.

Variations of bacterial spectrum and increasing antibiotic resistance necessitate selection of proper empiric antibiotic therapy against suspected infection. Study patients were treated with a five-day course of amoxicillin-clavulanic acid. Patients were treated with intravenous antibiotics during admission and were discharged with oral antibiotics to complete a five-day course of treatment. A large variety of antibiotic treatments for hand infections are suggested in the literature, ranging from cloxacillin, cefazolin, vancomycin, clindamycin, trimethoprim-sulfamethoxazole, doxycycline, meropenem, imipenem, amoxicillin-clavulanic acid, and piperacillin-tazobactam. ${ }^{5,12,17,26}$ Similar to other studies, this study showed good sensitivity of Staphylococcus aureus to amoxicillin-clavulanic acid, ${ }^{6,27}$ cefazolin, clindamycin, erythromycin/azithromycin and trimethoprimsulfamethoxazole. As both Staphylococcus aureus and Streptococcus spp. were found to be the dominant bacteria in hand infections, and both were sensitive to amoxicillinclavulanic acid, we therefore recommend amoxicillinclavulanic acid as empiric antibiotic treatment for hand sepsis in our catchment area.

\section{Conclusion}

In this study, patients with spontaneous infection were usually infected with Staphylococcus aureus, while patients with penetrating trauma, blunt trauma or insect bites were susceptible to both Staphylococcus aureus and Streptococcus spp. Patients with HIV had higher positive cultures for Staphylococcus aureus compared to other bacterial strains, whereas patients with diabetes mellitus had a higher incidence of Streptococcus spp. culture.

Diabetes as comorbidity seems to have the strongest effect on several metrics of severity, including a need for multiple procedures, amputations, skin grafts and a prolonged hospital stay.

Although, in this study, diverse microbial strains are harboured in hand sepsis in diabetics, the most common organism cultured is Streptococcus spp, followed by Staphylococcus aureus. The present study revealed that both the strains were sensitive to amoxicillin-clavulanic acid. Moreover, due to the increasing resistance of these strains to 
penicillin/ampicillin and cloxacillin, the current best option as empiric antibiotic treatment of hand sepsis in this clinical setting is amoxicillin-clavulanic acid.

\section{Acknowledgements}

Inkosi Albert Luthuli Central Hospital for permission to conduct this study. Mr PJ Naude for statistical assistance. Ms L Sewnarain for reviewing this article.

\section{Conflict of interest}

The authors declare no conflict of interest.

\section{Funding source}

None.

\section{Ethical approval}

The Biomedical Research Ethics Committee (BREC) of the University of KwaZulu-Natal approved the research (BE 588/17) and permission from Inkosi Albert Luthuli Hospital.

\section{ORCID}

$M$ van der Vyver (iD https://orcid.org/0000-0001-8384-5970 A Madaree (iD https://orcid.org/0000-0001-8661-0288

\section{REFERENCES}

1. Flevas DA, Syngouna S, Fandridis E, Tsiodras S, Mavrogenis AF. Infections of the hand - an overview. EFORT Open Rev. 2019;4:183-93.

2. Admas A, Gelaw B, Tessema B, Worku A, Melese A. Proportion of bacterial isolates, their antimicrobial susceptibility profile and factors associated with puerperal sepsis among post-partum/aborted women at referral hospital in Bahir Dar, Northwest Ethiopia. Antimicrob Resist Infect Control. 2020;9:14.

3. Arsalan-Wemer A, Grisar P, Sauerbier M. Risk factors for reoperation in primary hand infections - a multivariate analysis. Arch Orthop Trauma Surg. 2020;140(2):283-8.

4. McDonald LS, Bavaro MF, Hofmeister EP, Kroonen LT. Hand infections. J Hand Surg Am. 2011;36(8):1403-12.

5. Greyling J, Visser E, Elliot E. Bacteriology and epidemiology of hand infections. SA Orthop J. 2012;11(1):57-61.

6. Clark D. Common acute hand infections. Am Fam Physician. 2003;68(11):2167-76.

7. Klein M, Chang J. Hand infections. In: Greer SE, Benhaim P, Lorenz HP, Chang J, Hedrick MH, editors. Handbook of plastic surgery. United Kingdom: Taylor \& Francis; 2004. p. 631-36.

8. Ong Y, Levin L. Hand infections. Plast Reconstr Surg. 2009;124(4):225e-33e.

9. Patel D, Emmanuel N, Stevanovic M, et al. Hand infections - anatomy, types and spread of infection, imaging findings, and treatment options. Radio Graphics. 2014;34(7):1968-86.

10. David W, Roderick D, Erman M, Jane V. OSH hand surgery. In: David W, Dunn R, Melikyan E, Vadher J, editors. Specialist handbooks in surgery hand surgery. Oxford, UK: Oxford University Press; 2009. p. 275-80.

11. Chang J, Neligan PC. Plastic surgery: volume six - Hand and upper limb. 4th ed. London: Elsevier; 2017.

12. Stevanovic MV, Sharpe F. Acute infections. In: Wolfe SW, editor. Green's operative hand surgery. 6th ed. Philadelphia, US: Elsevier; 2011.

13. Shisana O, Rehle T, Simbayi LC, et al. South African National HIV prevalence, incidence and behaviour survey. Cape Town: HSRC Press; 2014.

14. Barry AL, Garcia F, Thrupp LD. An improved single-disk method for testing the antibiotic susceptibility of rapidlygrowing pathogens. Am J Clin Pathol. 1970;53:149-58.

15. Verhoef H, Marais LC, Ryan PV, Rollinson PD. The risk of early complications in patients with hand infections. SA Orthop J. 2020;19(2):97-102.

16. Prokosch V, Gatzioufas Z, Thanos S, Stupp T. Microbiological findings and predisposing risk factors in corneal ulcers. Graefes Arch Clin Exp Ophthalmol. 2012;250:369-74.

17. Dan M, Marien GJ, Sand C. Susceptibility of group B streptococci to cloxacillin, methicillin and cefamandole. J Antimicrob Chemother. 1983;11(1):89-92.

18. Houshian S, Seyedipour S, Wedderkopp N. Epidemiology of bacterial hand infections. Int J Infect Dis. 2006;10(4):315-9.

19. Turow A, Palapitige B, Kim SW, et al. Hand infection patients presenting to an orthopaedic unit - an audit of incidence and demographics at a rural hospital. Aust J Rural Health. 2016;24:48-53.

20. Heitmann C, Scott Levin L. Acute infections of the upper extremity. In: McCarthy JG, Galiano RD, Boutros SG, editors. Current therapy in plastic surgery. Philadelphia, US: WB Saunders; 2006. p. 640-6. https://doi.org/10.1016/B9780-7216-0000-0.50088-8.

21. Ince B, Dadaci $M$, Arslan A, et al. Factors determining poor prognostic outcomes following diabetic hand infections. Pak J Med Sci. 2015;31(3):532-7.

22. Duma MTN, Marais LC. Early complications of human bites to the hand in HIV positive patients. SA Orthop J. 2016;15(4):53-7.

23. Pang HN, Teoh LC, Yam AKT, et al. Factors affecting the prognosis of pyogenic flexor tenosynovitis. J Bone Joint Surg Am. 2007;89:1742-8.

24. Khamnuan $\mathrm{P}$, Chongruksut $\mathrm{W}$, Jearwattanakanok $\mathrm{K}$, Patumanond J, Tantraworasin A. Necrotizing fasciitis epidemiology and clinical predictors for amputation. Int J Gen Med. 2015;8:195-202.

25. Nawijn F, Verhiel SH, Lumn KN, et al. Factors associated with mortality and amputation caused by necrotizing soft tissue infections of the upper extremity - a retrospective cohort study. World J Surg. 2020;44:730-40.

26. Fitzgibbons PG, Weiss AP. Hand manifestations of diabetes mellitus. J Hand Surg Am. 2008;33(5):771-5.

27. Chambers HF. The changing epidemiology of Staphylococcus aureus? Emerg Infect Dis. 2001;7(2):178-82. 